\title{
Recent advances in the understanding and management of rosacea
}

\author{
Uwe Wollina
}

\author{
Address: Department of Dermatology and Allergology, Academic Teaching Hospital Dresden-Friedrichstadt, \\ Friedrichstrasse 41, 01067 Dresden, Germany \\ Email: wollina-uw@khdf.de \\ FI000Prime Reports 2014, 6:50 (doi:10.12703/P6-50) \\ All F1000Prime Reports articles are distributed under the terms of the Creative Commons Attribution-Non Commercial License (http:// \\ creativecommons.org/licenses/by-nc/3.0/legalcode), which permits non-commercial use, distribution, and reproduction in any medium, \\ provided the original work is properly cited. \\ The electronic version of this article is the complete one and can be found at: http://fl000.com/prime/reports/m/6/50
}

\begin{abstract}
Rosacea is a chronic relapsing inflammatory facial dermatosis. There are several known triggers but the pathogenesis remains unknown. Recent achievements in understanding this disease point to the importance of skin-environmental interactions. This includes physical and chemical factors, but also microbial factors. The impairment of the skin barrier function and the activation of the innate immune defences are major and connected pathways contributing to an ongoing inflammatory response in the affected skin. This becomes modulated by endogenous factors like neurovascular, drugs, and psychological factors. These factors offer new therapeutic targets for rosacea treatment. There is a broader range of anti-inflammatory compounds available with a favourable safety record. Only recently have persistent erythema and flushing been addressed by new drug formulations.
\end{abstract}

\section{Introduction}

Rosacea is a common inflammatory facial dermatosis. It affects mainly adults, but rare cases during childhood and adolescence have been observed. The prevalence of rosacea is highest among Indo-Eurasians [1]. In Europe, there is an increasing prevalence from South to North: in Germany prevalence is $2.2 \%$, in Sweden $10 \%$, and in Estonia 22\% [2-4]. There are some gender differences, since rosacea usually starts earlier among females and rhinophyma is almost exclusively seen among males. Ocular involvement is the most common extracutaneous manifestation and affects 6 to $50 \%$ of patients with rosacea [5]. In a retrospective investigation from Tunisia, the following frequency was observed in an outpatient dermatologic clinic: erythematotelangiectatic type $(12 \%)$, papulopustular rosacea (69\%), and rhinophyma $(3.7 \%)[6]$. This may vary considerably with the type of study and region.

Rosacea is accompanied by stinging and burning sensations of the affected skin. Redness, scaling, pustulation, and phymatous growth are other possible manifestations. Rosacea also has a negative impact on quality of life through stigmatizing feelings and anxiety of patients [7].
Rosacea patients also have an increased risk for cardiovascular disease [8]. In a rapidly growing elderly population in Western countries, rosacea will become an increasing health problem [9].

Although the pathogenesis of rosacea is unknown, there have been a number of recent advances in understanding the disease. Major achievements will be described together with their potential for better treatment for rosacea patients.

\section{Classification of rosacea}

Rosacea has been classified into four subtypes [10]. Subtype 1, or erythematotelangiectatic rosacea, is defined by the presence of flushing and central facial erythema. Additional possible features are edema, stinging and burning sensations, as well as roughness or scaling. Subtype 2, or papulopustular rosacea, is defined by persistent erythema and transient papules or pustules. It is a more inflammatory rosacea subtype. Subtype 3, or phymatous rosacea, presents with thickening skin, irregular surface nodularities, and enlargement of affected areas. Areas affected by phymatous rosacea are chin, forehead, cheeks, ears, and nose, with nose or rhinophyma being the 
most common phenotype by far with a clear male predominance. Ocular rosacea is defined as subtype 4.

Erythema in rosacea can be subdivided into (a) sole erythema; (b) erythema with telangiectasias; (c) erythema with edema; and (d) erythema with inflammatory papules and nodules. It is of great therapeutic importance to differentiate the perilesional erythema of inflammatory lesions from the diffuse facial erythema [11].

\section{Rosacea and the epidermal barrier function}

The clinical observation of a tight association of rosacea with sensitive skin has led to the concept of a disturbed epidermal barrier function in rosacea. A lower irritant threshold corresponds to higher transepidermal water loss (TEWL) and lower stratum corneum hydration. In contrast to atopic dermatitis, the epidermal barrier deterioration in rosacea remains restricted to facial skin [12]. Increased basal TEWL activates certain epidermal proteases, in particular stratum corneum serine proteases [13].

The activation of proteases is the link between impaired barrier function and inflammation in rosacea. The trypsinlike serine protease kallikrein 5 is increased in lesional rosacea. Kallikrein 5 is responsible for the cleavage and activation of cathelicidin LL37-an antimicrobial peptide of skin. In rosacea lesions, both LL-37 and its proteolytic fragments are found in larger amounts than normal [14].

\section{Rosacea and the innate immune system}

Toll-like receptors (TLRs) are pattern recognition receptors tightly bound to innate immunity. TLR2 expression is increased in rosacea. This leads to a higher susceptibility of rosacea skin to innate immune stimuli. By activating TLR2, the production of serine proteases in keratinocytes is stimulated [15].

Antimicrobial peptides are part of the innate immune system of human skin. In rosacea, LL-37 levels are remarkably increased-at least in part by TLR2. This may exert various effects related to rosacea pathogenesis and clinical presentation. LL-37 is antimicrobial, pro-inflammatory and angiogenic [16-18].

Various chemokines and cytokines seem to be involved in the inflammatory cutaneous changes of rosacea. Members of the tumor growth factor (TGF) family have been linked to phymatous rosacea. In particular, TGF- $\beta 1$ and TGF- $\beta 2$ and the TGF- $\beta$ receptor type II are overexpressed in phymatous rosacea [19]. A crucial step in chemoattraction of neutrophils is the chemokine CXCL8 induced by LL-37 [20]. Eventually, formation of sterile pustules of subtype 2 rosacea occurs.

\section{Neurogenic inflammation and vascular changes in rosacea}

The prolonged flush and persistent erythema are hallmarks of disturbed facial blood flow. Vascular endothelial growth factor (VEGF) is present in epidermis and is expressed by infiltrating cells. VEGF receptor-ligand binding contributes to the vascular changes and cellular infiltration that occurs in rosacea [21]. Another receptor related to local blood flow, vasoactive intestinal peptide (VIP) receptor, is increased in cutaneous blood vessels of phymatous rosacea and in serum $[22,23]$.

Substance P has also been linked to rosacea [24]. This neuropeptide is involved in local blood flow regulation and induces mast cell degranulation leading to increased levels of pro-inflammatory chemokines and cytokines, such as CXCL8, tumor necrosis factor (TNF)- $\alpha$, and interleukin (IL)-3 [25].

Rosacea skin has a significantly lower heat pain threshold than normal skin. Since transient receptor potential ion channels of the vanilloid type (TRPV) 1 , and ankyrin 1 (TRPA1) function as cellular sensors for phenomena such as cold and heat, and have a prominent role in pain sensation and inflammation, they are possibly involved in rosacea. The activation of such receptors may increase the release of substance P [26]. A recent study demonstrated that dermal immunostaining of TRPV2 and TRPV3 and gene expression of TRPV1 is significantly increased in subtype 1 rosacea. Subtype 2 rosacea demonstrated enhanced immunoreactivity for TRPV2, TRPV4, and also of TRPV2 gene expression. In subtype 3, rosacea dermal immunostaining of TRPV3 and TRPV4 and gene expression of TRPV1 and TRPV 3 was enhanced, whereas epidermal TRPV2 staining was decreased. These data argue for an involvement of TRPVs in rosacea. The different TRPVs have an impact on local immune function, vascular regulation, nociception, and epidermal barrier integrity [27].

\section{Ultraviolet light radiation and rosacea}

Ultraviolet light radiation (UVR) is a well-known trigger of rosacea. UVR induces the formation of reactive oxygen species (ROS) in skin [28]. TLR2-ROS interaction leads to increased levels of CXCL8 among other chemokines and cytokines. Furthermore, myeloid differentiation factor MyD88-an adaptor molecule for TLRs-is overexpressed in UV-irradiated and chronic photodamaged skin. It upregulates both IL-6 and matrix metalloproteinase-1 [29].

\section{Demodex mites and rosacea}

The human mites Demodex folliculorum and Demodex brevis are the cause of demodicosis, a disease that can imitate a 
variety of other dermatoses, including rosacea. Therefore, it is important to differentiate primary demodicosis from rosacea in order to choose the right treatment option [30].

There has been a long debate on the role of Demodex mites in rosacea itself. The prevalence of Demodex mites in rosacea patients has been estimated to be as high as $60 \%$ (clinically) and 80\% (in skin biopsies), with Demodex folliculorum as the dominant infestation [31,32]. Increased Demodex density in rosacea is considered to be an aggravating factor but not a causative one $[33,34]$.

\section{Treatment of rosacea}

The classical approach of rosacea therapy has focused on the inflammatory nodules, pustules and papules. The two most effective topical drugs for rosacea subtype 2 are metronidazole (as gels or ointments) and azelaic acid gel-recently also available as foam [35].

Azelaic acid $15 \%$ gel is a topical therapeutic option for subtype 2 rosacea. Azelaic acid reduces ROS, inhibits kallikrein 5 and increases serine protease activity [36,37]. Azelaic acid $15 \%$ gel is a US Food and Drug Administration (FDA) approved drug for treating inflammatory papules and pustules in mild to moderate rosacea, due to its anti-inflammatory, anti-oxidant and anti-microbial properties. A newly developed azelaic acid 15\% foam formula demonstrated a statistically significant advantage compared to the gel formula in a randomized, double blinded trial with 486 patients over a 12 weeks period, when applied twice daily [38]. A noticeable advantage with azelaic acid foam is that adverse effects (including burning, stinging or itching) were only observed in $10.6 \%$ of these participants while $38 \%$ of patients using azelaic acid gel had experienced side effects in an earlier trial [39]. The advancement from gel to foam formula may promote more rapid drug penetration and greater total absorption. Foam is becoming increasingly popular with patients due to its ease of application and spread, faster drying time and reduced density, resulting in a higher rate of patient compliance.

Topical metronidazole is of comparable efficacy to azelaic acid gel. It is available as $0.75 \%$ gel, $1 \%$ gel and $1 \%$ ointment. It works as an oxygen scavenger, thereby decreasing ROS and inhibiting neutrophils [40].

Recently, topical ivermectin $1 \%$ has been evaluated in two randomized, double-blind, controlled studies for 12 weeks. In both trials ivermectin $1 \%$ was superior to the vehicle alone in terms of anti-inflammatory activity and safety [41]. Ivermectin is an anti-helminthic drug and a ligand of farnesoid $\mathrm{X}$ receptor, which seems to decrease the density of Demodex mites in rosacea skin [42].

Topical calcineurin inhibitors, which inhibit both T-lymphocyte signal transduction and IL-2 transcription, such as pimecrolimus $1 \%$ cream and tacrolimus ointment $0.03 \%$ or $0.1 \%$, can treat rosacea [43].

Kim et al. (2011) investigated the efficacy and safety of 1\% pimecrolimus cream for the treatment of rosacea in a 4 -week, single-center, open-label study of $1 \%$ pimecrolimus $(\mathrm{n}=30)$. The 26 patients who completed the study experienced significantly reduced rosacea clinical scores and mexameter-measured erythema index. The most common side-effects were transient local irritations [44]. Koca et al. (2010) compared the effectiveness of $1 \%$ pimecrolimus to metronidazole on 48 patients in an open-labeled, randomized, single-center study and found them equally effective [45]. Prospective randomized, multi-center studies have not yet been performed.

Topical calcineurin inhibitors can induce rosacea-like dermatitis and flush, often affecting the cheeks, perioral/ perinasal area and forehead/glabellar area [46]. These observations may be explained by propagation of demodicosis due to topical immunosuppression, and drug interactions with alcoholic beverages $[46,47]$.

Skin care products moisturizing the skin, and thereby reducing stinging, burning and the feeling of tightness of skin, are an adjuvant measure. The applications for such adjuvant treatments are subtypes 1 and $2[48,49]$. Some open questions remain. What are the essentials for skin care products in rosacea? Are there cosmetic ingredients with seriously proven efficacy in such patients? So far, no head-to-head studies have been performed to address these questions. Although UVR is a trigger factor for rosacea, there have been no systematic investigations of sun protection.

Oral tetracyclines, like doxycyline and minocycline, have been a cornerstone of systemic treatment in rosacea subtypes 2 and 4 . In recent years, the need for an antibiotic effect has been questioned, with regards to rosacea. This has led to the development of a modifiedrelease formulation of anti-inflammatory doxycycline $40 \mathrm{mg}$ given once daily. Time-released formulations of minocycline are also available. It has been shown that tetracyclines decrease matrix metalloproteinase activity involved in kallikrein activation and also act as oxygen scavengers [50,51]. Furthermore, epidermal barrier function improves after tetracycline therapy, as measured by epidermal hydration [52]. 
Recently, azithromycin has been used to effectively treat ocular and cutaneous rosacea, inhibiting a cluster of proinflammatory cytokines (including IL-1, IL-6, IL-8, IL-10, and TNF- $\alpha$ ), down-regulating the expression of nuclear factor (NF) kappaB, and inhibiting neutrophil chemoattractant leukotriene B4. Bakar et al. (2009) showed that patients who received $500 \mathrm{mg}$ oral azithromycin on three consecutive days, weekly for four weeks, showed significant improvements in their ocular symptoms [53]. Doan et al. (2013) further substantiated the effectiveness of azithromycin when their study was performed using 1.5\% azithromycin eye drops with 16 children on a median 11 months follow-up. In a single case that did not completely respond, cyclosporine $2 \%$ eye drops were added. These investigators stopped azithromycin treatment after 4 to 10 months and there were no recurrences during follow-up [54].

During pregnancy, acute rosacea flare-ups may occur. Fuentelsaz et al. described a pregnant woman with rosacea fulminans who was successfully treated after a 3-month tapering course of azithromycin [55], which has been classified as safer than tetracyclines. Whether the dosage, and dosing schedule, of azithromycin may be lowered such that it is sub-antimicrobial but is effective as an anti-inflammatory agent is unclear.

There is some evidence that a combination of topical and systemic therapy might provide better and faster results in initiation therapy, whereas topical treatment is preferred for maintenance [56].

All previous medical treatments failed to improve erythema. Diffuse central facial erythema of rosacea is often chronically present, even in patients who are not experiencing acute flare-ups. Erythema with telangiectasias may improve by laser or intense pulsed light (IPL) therapies [57].

Selective $\alpha$-adrenergic inhibitors demonstrate vasoconstrictive efficacy. There is extensive clinical experience with brimonidine for glaucoma therapy and with oxymetazoline in rhinitis. Topical $\alpha$-adrenoreceptor agonists brimonidine and oxymetazoline are now being used to treat rosacea, due to the fact that they target $\alpha$-adrenoreceptors in the smooth muscle sheath around the cutaneous blood vessels. However, $\alpha$-agonists have no effect on capillaries and telangiectasias, since they do not contain a smooth muscle layer. Nonetheless, their effects on pre-capillary arterioles do provide significant reduction in the erythema of rosacea [58].

Two phase II trials with topical $\alpha 2$-adrenergic inhibitor brimonidine tartrate, involving 122 and 262 rosacea patients, demonstrated a dose-dependent decrease of facial erythema without rebound or tachyphylaxis. The topical treatment was well tolerated with only mild and temporary adverse effects. The most efficacious formulation was $0.5 \%$ brimonidine tartrate leading to significant improvement in $75.5 \%$ of patients. Even at the final point of evaluation, the intensity of erythema did not return to the initial baseline level [59]. Systemic exposure to brimonidine tartrate was lower in $0.5 \%$ gel formulation than in ophthalmic solution [60]. A one-year openlabel study confirmed the consistent efficacy and tolerability of topical treatment, which has gained FDA approval [61]. The resistance to $\alpha$-adrenergic blockage in about $30 \%$ of rosacea patients with moderate to severe erythema is not yet understood. It is possible that other neurovascular mechanisms may be involved.

The $\alpha 1$-selective inhibitor oxymetazoline has been used off-label as $0.05 \%$ solution in some cases, but results of clinical trials have yet not been published [62].

Ocular rosacea has a significant impact on quality of life. The treatment of ocular rosacea is based on systemic antiinflammatory drug therapy, topical therapy with artificial tears, and eyelid hygiene. Once-daily low dose (40 mg) doxycycline is a safe and effective treatment [63]. Cyclosporine $\mathrm{A}$ is a calcineurin inhibitor that possesses anti-inflammatory properties. It is capable of inhibiting lipopolysaccharide-induced NF-kappaB activation and acts as an uncompetitive inhibitor of the chymotrypsinlike activity of the $20 \mathrm{~S}$ proteasome in vitro [64]. Cyclosporine A $0.05 \%$ ocular emulsion is effective in treating dry eye caused by autoimmune connective tissue disease, and has potential in ocular rosacea as well [65]. Schechter et al. (2009) successfully demonstrated the efficacy of a $0.05 \%$ cyclosporine A ophthalmic emulsion twice daily for 3 months, on ocular rosacea, in a doubleblinded study of 37 patients [66].

A recent alternative to systemic low-dose tetracyclines is topical azithromycin eye drops. The major adverse effect is mild burning after instillation. In a study enrolling 37 patients with ocular rosacea, the necessary treatment time was shorter when compared to systemic doxycycline [67].

There are currently several drugs to treat rosacea in ongoing clinical trials (see Table 1 ). New potential rosacea drugs may be targeting the TRPVs.

\section{Conclusion}

Although we are far away from a complete understanding of the complex pathogenesis of rosacea, there has been much progress in recent times. Treatment tailored to 
Table I: Registered clinical trials for rosacea with medical drugs [68]

\begin{tabular}{ll}
\hline NCT-No. & Drug(s) \\
\hline NCTOII25930 & atralin gel (tretinoin 0.5\%) \\
NCTOI04555I & apremilast \\
NCTOII3499I & topical minocyline foam \\
\hline
\end{tabular}

specific rosacea subtypes has been incorporated in clinical guidelines. The importance of differential diagnosis of perilesional and persistent facial erythema is evident. Rosacea therapy has become effective, safer and for the first time includes a medical approach to improve persistent erythema.

With further investigations into the biochemical pathways specifically uncovering the key mediators of inflammation, more effective and potent drugs can control the condition, change its natural history, and perhaps induce a remission or cure.

\section{Abbreviations}

FDA, US Food and Drug Administration; IL, interleukin; IPL, intense pulsed light; NF, nuclear factor; ROS, reactive oxygen species; TEWL, transepidermal water loss; TGF, tumor growth factor; TLR, toll-like receptor; TNF, tumor necrosis factor; TRP, transient receptor potential ion channel; TRPV, transient receptor potential ion channel of the vanilloid type; UVR, ultraviolet light radiation; VEGF, vascular endothelial growth factor.

\section{Disclosures}

The author declares that he has no disclosures.

\section{References}

I. Wollina U, Verma SB: Rosacea and rhinophyma: not curse of the Celts but Indo Eurasians. J Cosmet Dermatol 2009, 8:234-5.

2. Berg M, Lidén S: An epidemiological study of rosacea. Acta Derm Venereol 1989, 69:419-23.

\section{FlOOOPrime}

\section{RECOMMENDED}

3. Schaefer I, Rustenbach SJ, Zimmer L, Augustin M: Prevalence of skin diseases in a cohort of 48,665 employees in Germany. Dermatology (Basel) 2008, 217:169-72.

\section{FlOOOPrime RECOMMENDED}

4. Abram K, Silm H, Ona M: Prevalence of rosacea in an Estonian working population using a standard classification. Acta Derm Venereol 2010, 90:269-73.

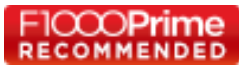

5. Böhm D, Schwanitz P, Stock Gissendanner S, Schmid-Ott G, Schulz W: Symptom severity and psychological sequelae in rosacea: Results of a survey. Psychol Health Med 2013; in press.

6. Khaled A, Hammami H, Zeglaoui F, Tounsi J, Zermani R, Kamoun MR, Fazaa B: Rosacea: 244 Tunisian cases. Tunis Med 20I0, 88:597-60I.
7. Duman N, Ersoy Evans S, Atakan N: Rosacea and cardiovascular risk factors: a case control study. J Eur Acad Dermatol Venereol 2013; in press.

8. Powell FC: Clinical practice. Rosacea. N Engl J Med 2005, 352:793-803.

9. Wollina U: Rosacea and rhinophyma in the elderly. Clin Dermatol 20II, 29:6I-8.

10. Wilkin J, Dahl M, Detmar M, Drake L, Feinstein A, Odom R, Powell F: Standard classification of rosacea: Report of the National Rosacea Society Expert Committee on the Classification and Staging of Rosacea. J Am Acad Dermatol 2002, 46:584-7.

II. Del Rosso, James Q: Advances in understanding and managing rosacea: part 2: the central role, evaluation, and medical management of diffuse and persistent facial erythema of rosacea. J Clin Aesthet Dermatol 2012, 5:26-36.

12. Darlenski R, Kazandjieva J, Tsankov N, Fluhr JW: Acute irritant threshold correlates with barrier function, skin hydration and contact hypersensitivity in atopic dermatitis and rosacea. Exp Dermatol 2013, 22:752-3.

\section{FlOOOPrime}

RECOMMENDED

13. Voegeli R, Rawlings AV, Doppler S, Schreier T: Increased basal transepidermal water loss leads to elevation of some but not all stratum corneum serine proteases. Int J Cosmet Sci 2008, 30:435-42.

14. Meyer-Hoffert U, Schröder J: Epidermal proteases in the pathogenesis of rosacea. J Investig Dermatol Symp Proc 20I I, 15:16-23.

15. Larrick JW, Hirata M, Zhong J, Wright SC: Anti-microbial activity of human CAP I 8 peptides. Immunotechnology 1995, I:65-72.

16. Koczulla R, Degenfeld G von, Kupatt C, Krötz F, Zahler S, Gloe T, Issbrücker K, Unterberger P, Zaiou M, Lebherz C, Karl A, Raake P, Pfosser A, Boekstegers P, Welsch U, Hiemstra PS, Vogelmeier C, Gallo RL, Clauss $M$, Bals R: An angiogenic role for the human peptide antibiotic LL-37/hCAP- I 8. J Clin Invest 2003, I I I: I 665-72.

17. Dorschner RA, Pestonjamasp VK, Tamakuwala S, Ohtake T, Rudisill J, Nizet V, Agerberth B, Gudmundsson GH, Gallo RL: Cutaneous injury induces the release of cathelicidin anti-microbial peptides active against group A Streptococcus. J Invest Dermatol 200I, I I7:91-7.

18. Payne WG, Wang X, Walusimbi M, Ko F, Wright TE, Robson MC: Further evidence for the role of fibrosis in the pathobiology of rhinophyma. Ann Plast Surg 2002, 48:64I-5.

19. Yamasaki K, Kanada K, Macleod DT, Borkowski AW, Morizane S, Nakatsuji T, Cogen AL, Gallo RL: TLR2 expression is increased in rosacea and stimulates enhanced serine protease production by keratinocytes. J Invest Dermatol 201 I, I31:688-97.

20. Zhang J, Xu X, Rao NV, Argyle B, McCoard L, Rusho WJ, Kennedy TP, Prestwich GD, Krueger G: Novel sulfated polysaccharides disrupt cathelicidins, inhibit RAGE and reduce cutaneous inflammation in a mouse model of rosacea. PLOS ONE 2011, 6:el6658.

\section{FlOOOPrime}

\section{RECOMMENDED}

21. Smith JR, Lanier VB, Braziel RM, Falkenhagen KM, White C, Rosenbaum JT: Expression of vascular endothelial growth factor and its receptors in rosacea. $\mathrm{Br} J$ Ophthalmol 2007, 91 1:226-9.

\section{FlOOOPrime} RECOMMENDED

22. Wollina U: Rhinophyma-unusual expression of simple-type keratins and SIOOA in sebocytes and abundance of VIP receptor-positive dermal cells. Histol Histopathol I 996, I I: I I I-5.

23. Lange D, Funa K, Ishisaki A, Bauer R, Wollina U: Autocrine endothelial regulation in brain stem vessels of newborn piglets. Histol Histopathol 1999, 14:82 I-5.

24. Powell FC, Corbally N, Powell D: Substance $\mathbf{P}$ and rosacea. J Am Acad Dermatol 1993, 28:132-3. 
25. Kulka M, Sheen CH, Tancowny BP, Grammer LC, Schleimer RP: Neuropeptides activate human mast cell degranulation and chemokine production. Immunology 2008, I 23:398-4I0.

26. Aubdool AA, Brain SD: Neurovascular aspects of skin neurogenic inflammation. J Investig Dermatol Symp Proc 20 I I, I 5:33-9.

27. Sulk M, Seeliger S, Aubert J, Schwab VD, Cevikbas F, Rivier M, Nowak P, Voegel J], Buddenkotte J, Steinhoff M: Distribution and expression of non-neuronal transient receptor potential (TRPV) ion channels in rosacea. J Invest Dermatol 20I2, I32:I253-62.

\section{FlOOOPrime
RECOMMENDED}

28. Thiele JJ, Schroeter C, Hsieh SN, Podda M, Packer L: The antioxidant network of the stratum corneum. Curr Probl Dermatol 200I, 29:26-42.

29. Yamasaki K, Gallo RL: The molecular pathology of rosacea. J Dermatol Sci 2009, 55:77-8I.

30. Chen W, Plewig G: Human demodicosis: revisit and a proposed classification. Br J Dermatol 2014; in press.

31. Yücel A, Yilmaz M: Rosacea Ön Tanılı Hastalarda Demodex folliculorum ve Demodex brevis Yaygınlığının Araştırılması. Turkiye Parazitol Derg 2013, 37:195-8.

\section{FlOOOPrime RECOMMENDED}

32. Ríos-Yuil JM, Mercadillo-Perez P: Evaluation of Demodex folliculorum as a Risk Factor for the Diagnosis of Rosacea In Skin Biopsies. Mexico's General Hospital (I975-2010). Indian J Dermatol 2013, 58:157.

\section{FlOOOPrime}

\section{RECOMMENDED}

33. Powell FC: Rosacea and the pilosebaceous follicle. Cutis 2004, 74:9-12, 32-4.

34. Holmes AD: Potential role of microorganisms in the pathogenesis of rosacea. J Am Acad Dermatol 2013, 69:1025-32.

35. van Zuuren E J, Kramer SF, Carter BR, Graber MA, Fedorowicz Z: Effective and evidence-based management strategies for rosacea: summary of a Cochrane systematic review. $\mathrm{Br} J$ Dermatol 2011, I65:760-81.

\section{FlOOOPrime}

\section{RECOMMENDED}

36. Gollnick H, Layton A: Azelaic acid I5\% gel in the treatment of rosacea. Expert Opin Pharmacother 2008, 9:2699-706.

37. Coda AB, Hata T, Miller J, Audish D, Kotol P, Two A, Shafiq F, Yamasaki K, Harper JC, Del Rosso, James Q, Gallo RL: Cathelicidin, kallikrein 5 , and serine protease activity is inhibited during treatment of rosacea with azelaic acid $15 \%$ gel. J Am Acad Dermatol 2013, 69:570-7.

38. Draelos ZD, Elewski B, Staedtler G, Havlickova B: Azelaic acid foam $15 \%$ in the treatment of papulopustular rosacea: a randomized, double-blind, vehicle-controlled study. Cutis 2013, 92:306-I7.

\section{FlOOOPrime \\ RECOMMENDED}

39. Thiboutot D, Thieroff-Ekerdt R, Graupe K: Efficacy and safety of azelaic acid (15\%) gel as a new treatment for papulopustular rosacea: results from two vehicle-controlled, randomized phase III studies. J Am Acad Dermatol 2003, 48:836-45.

\section{FlOOOPrime}

\section{RECOMMENDED}

40. Narayanan S, Hünerbein A, Getie M, Jäckel A, Neubert Reinhard H H: Scavenging properties of metronidazole on free oxygen radicals in a skin lipid model system. J Pharm Pharmacol 2007, 59: I I 25-30.

41. Stein L, Kircik L, Fowler J, Tan J, Draelos Z, Fleischer A, Appell M, Steinhoff M, Lynde C, Liu H, Jacovella J: Efficacy and safety of ivermectin $1 \%$ cream in treatment of papulopustuIar rosacea: results of two randomized, double-blind, vehiclecontrolled pivotal studies. J Drugs Dermatol 2014, I3:3 I6-23.

\section{FlOOOPrime
RECOMMENDED}

42. Jin L, Feng X, Rong H, Pan Z, Inaba Y, Qiu L, Zheng W, Lin S, Wang R, Wang Z, Wang S, Liu H, Li S, Xie W, Li Y: The antiparasitic drug ivermectin is a novel FXR ligand that regulates metabolism. Nat Commun 2013, 4:1937.

43. Wollina $U$ : The role of topical calcineurin inhibitors for skin diseases other than atopic dermatitis. Am J Clin Dermatol 2007, 8: $157-73$.

44. Kim M, Kim G, Park H, Kim H, Chin H, Kim S, Kim B, Ko H: Pimecrolimus I\% cream for the treatment of rosacea. J Dermatol 201 I, 38: I135-9.

FlOOOPrime

RECOMMENDED

45. Koca R, Altinyazar HC, Ankarali H, Muhtar S, Tekin NS, Cinar S: A comparison of metronidazole I\% cream and pimecrolimus $1 \%$ cream in the treatment of patients with papulopustular rosacea: a randomized open-label clinical trial. Clin Exp Dermatol 2010, 35:251-6.

FlOOOPrime RECOMMENDED

46. Teraki Y, Hitomi K, Sato Y, Izaki S: Tacrolimus-induced rosacealike dermatitis: a clinical analysis of 16 cases associated with tacrolimus ointment application. Dermatology (Basel) 2012, 224:309-I4.

47. Ogunleye T, James WD: Ethanol-induced flushing with topical pimecrolimus use. Dermatitis 2008, I9:EI-2.

48. Wu JJ, Weinstein GD, Kricorian GJ, Kormeili T, McCullough JL: Topical kinetin $0.1 \%$ lotion for improving the signs and symptoms of rosacea. Clin Exp Dermatol 2007, 32:693-5.

49. Seite S, Benech F, Berdah S, Bayer M, Veyrat S, Segot E, Sakalikova M, Gibejova L, Zelenkova H: Management of rosacea-prone skin: evaluation of a skincare product containing Ambophenol, Neurosensine, and La Roche-Posay Thermal spring water as monotherapy or adjunctive therapy. J Drugs Dermatol 2013, I 2:920-4.

50. Kanada KN, Nakatsuji T, Gallo RL: Doxycycline indirectly inhibits proteolytic activation of tryptic kallikrein-related peptidases and activation of cathelicidin. J Invest Dermatol 2012, I32: |435-42.

5I. Scheinfeld N, Berk T: A review of the diagnosis and treatment of rosacea. Postgrad Med 2010, I 22:139-43.

52. Ní Raghallaigh S, Powell FC: Epidermal hydration levels in rosacea patients improve after minocycline therapy. $\mathrm{Br} J$ Dermatol 2013.

53. Bakar O, Demircay Z, Toker E, Cakir S: Ocular signssymptoms and tear function tests of papulopustular rosacea patients receiving azithromycin. J Eur Acad Dermatol Venereol 2009, 23:544-9.

FlOOOPrime
RECOMMENDED

54. Doan S, Gabison E, Chiambaretta F, Touati M, Cochereau I: Efficacy of azithromycin $1.5 \%$ eye drops in childhood ocular rosacea with phlyctenular blepharokeratoconjunctivitis. J Ophthalmic Inflamm Infect 2013, 3:38. FlOOOPrime RECOMMENDED

55. Fuentelsaz V, Ara M, Corredera C, Lezcano V, Juberias P, Carapeto FJ: Rosacea fulminans in pregnancy: successful treatment with azithromycin. Clin Exp Dermatol 20I I, 36:674-6.

56. Bhatia ND, Del Rosso, James Q: Optimal management of papulopustular rosacea: rationale for combination therapy. J Drugs Dermatol 20 I 2, I I :838-44.

57. Tanghetti E, Del Rosso, James Q, Thiboutot D, Gallo R, Webster G, Eichenfield LF, Stein-Gold L, Berson D, Zaenglein A: Consensus recommendations from the American Acne \& Rosacea Society 
on the management of rosacea, part 4: a status report on physical modalities and devices. Cutis 2014, 93:7I-6.

58. Schwab VD, Sulk M, Seeliger S, Nowak P, Aubert J, Mess C, Rivier M, Carlavan I, Rossio P, Metze D, Buddenkotte J, Cevikbas F, Voegel J], Steinhoff $M$ : Neurovascular and neuroimmune aspects in the pathophysiology of rosacea. J Investig Dermatol Symp Proc 201 I, 15:53-62.

59. Fowler J, Jarratt M, Moore A, Meadows K, Pollack A, Steinhoff M, Liu Y, Leoni M: Once-daily topical brimonidine tartrate gel $\mathbf{0 . 5 \%}$ is a novel treatment for moderate to severe facial erythema of rosacea: results of two multicentre, randomized and vehicle-controlled studies. $\mathrm{Br}$ J Dermatol 20I2, I66:633-4I.

\section{FlOOOPrime} RECOMMENDED

60. Benkali K, Leoni M, Rony F, Bouer R, Fernando A, Graeber M, Wagner N: Comparative pharmacokinetics and bioavailability of brimonidine following ocular administration and dermal application of brimonidine tartrate ophthalmic solution and gel in subjects with moderate to severe facial erythema of rosacea. BrJ Dermatol 20I4; in press.

61. Moore A, Kempers S, Murakawa G, Weiss J, Tauscher A, Swinyer L, Liu H, Leoni M: Long-term safety and efficacy of once-daily topical brimonidine tartrate gel $0.5 \%$ for the treatment of moderate to severe facial erythema of rosacea: results of a I-year open-label study. J Drugs Dermatol 20|4, I3:56-6I.
62. Generali JA, Cada DJ: Oxymetazoline (topical): rosacea. Hosp Pharm 2013, 48:558-9.

63. Sobolewska B, Doycheva D, Deuter C, Pfeffer I, Schaller M, Zierhut M: Treatment of ocular rosacea with once-daily lowdose doxycycline. Cornea 2014, 33:257-60.

FlOOOPrime
RECOMMENDED

64. Meyer S, Kohler NG, Joly A: Cyclosporine $\mathbf{A}$ is an uncompetitive inhibitor of proteasome activity and prevents NF-kappaB activation. FEBS Lett 1997, 413:354-8.

65. Scheinfeld N: A review of deferasirox, bortezomib, dasatinib, and cyclosporine eye drops: possible uses and known side effects in cutaneous medicine. J Drugs Dermatol 2007, 6:352-5.

66. Schechter BA, Katz RS, Friedman LS: Efficacy of topical cyclosporine for the treatment of ocular rosacea. Adv Ther 2009, 26:65I-9.

\section{FlOOOPrime}

RECOMMENDED

67. Mantelli F, Di Zazzo A, Sacchetti M, Dianzani C, Lambiase A, Bonini S: Topical azithromycin as a novel treatment for ocular rosacea. Ocul Immunol Inflamm 2013, 2 I:37I-7.

68. ClinicalTrials.gov. [http://www.clinicaltrials.gov/] 